Perovskite-derived $\mathrm{Cs}_{2} \mathrm{SnCl}_{6}$-Silica Composites as Advanced Waste Forms for Chloride Salt Wastes

\author{
Kun Yang ${ }^{l}$, Weiguang Zhu, ${ }^{l}$ Brian J. Riley ${ }^{2}$, John D. Vienna, ${ }^{2}$ Dong Zhao, ${ }^{1}$ Jie Lian ${ }^{1 *}$ \\ 1. Department of Mechanical, Aerospace and Nuclear Engineering, Rensselaer Polytechnic Institute, \\ NY, US, 12180 \\ 2. $\quad$ Pacific Northwest National Laboratory, Richland, WA 99354 \\ *Corresponding author, Email: lianj@rpi.edu; Office: 518-276-6081; Fax: 518-276-6025
}

\author{
Number of Pages: 12 \\ Number of Tables: 2 \\ Number of Figures: 10 \\ Number of References: 2
}




\section{Thermal stability and decomposition}

Three different mass loss zones can be identified from the TGA profiles suggesting the stepwise decomposition process of the $\mathrm{Cs}_{2} \mathrm{SnCl}_{6}$. (Figure 1b) The initial decomposition of $\mathrm{Cs}_{2} \mathrm{SnCl}_{6}$ to $\mathrm{CsCl}$ and $\mathrm{SnCl}_{4}$ occurred from $570{ }^{\circ} \mathrm{C}$ to $670-690{ }^{\circ} \mathrm{C}$ with a simultaneous loss of $\mathrm{SnCl}_{4}$ due to its low boiling point $\left(114.2{ }^{\circ} \mathrm{C}\right) .{ }^{1}$ With the increasing of temperature, both $\mathrm{CsCl}$ and $\mathrm{SnCl}_{4}$ escaped accounting for the significant weight loss across the second stage. However, there is still minor weight loss above $800{ }^{\circ} \mathrm{C}$, which can be attributed to the decomposition of residual $\mathrm{SnCl}_{4}$ to $\mathrm{Sn}$ and $\mathrm{Cl}$ would be escaped from the matrix as $\mathrm{Cl}_{2}$. The elemental analysis of the post-TGA tested sample pellet is shown in Figure s6. Both the elemental mapping and EDS spot analysis on the $50 \mathrm{wt} \% \mathrm{Cs}_{2} \mathrm{SnCl}_{6}$-silica composite matrix post TGA test show the presence of Si and Sn without Cs and Cl. Interestingly, the final step weight loss occurred at higher temperatures with the increasing of silica ratio from $30 \mathrm{wt} \%$ to $70 \mathrm{wt} \%$ in the composite matrix (see the TGA curves shown in Figure 1b), which can be attributed to the high viscosity of the silica that can slow down the $\mathrm{Cl}_{2}$ diffusion under elevated temperatures. ${ }^{2}$

\section{Dual phase property of the as-sintered $\mathrm{Cs}_{2} \mathrm{SnCl}_{6}$-silica composite structures}

The crystalline phase structures and the surface micro-structure analysis can be seen in Figure s1s5. The SEM images (BSE mode) clearly denotes a dual-phase property for all the three composite structures with variable ratio of $\mathrm{Cs}_{2} \mathrm{SnCl}_{6}$. Moreover, the XRD profiles of the as-sintered composite structures as compared to the powder $\mathrm{Cs}_{2} \mathrm{SnCl}_{6}$ all demonstrate single phase of $\mathrm{Cs}_{2} \mathrm{SnCl}_{6}$ which can be clearly indexed by the standard PDF card.(Figure s1d) The elemental spot analysis, line scanning and elemental mappings for on the as-sintered composite structure further confirms the chemical stoichiometry which demonstrates the light colored region belongs to $\mathrm{Cs}_{2} \mathrm{SnCl}_{6}$ and dark color is silica. The line scanning clearly denotes the region enriched in $\mathrm{Cs}_{2} \mathrm{SnCl}_{6}$ is depleted in $\mathrm{Si}$ as shown in Figure $\mathrm{s} 3$-s5. The fracture surface of the $50 \mathrm{wt} \%$ composite structure clearly denotes a sandwich structure with $\mathrm{Cs}_{2} \mathrm{SnCl}_{6}$ being successfully encapsulated in the silica matrix in a highly consolidated structure layer by layer.

\section{Reference:}


[1] Scott S.M., Zhu W.G., Yao T.K., Vienna J.D, Ewing R.C., Lian J. The thermal stability and consolidation of perovskite variant $\mathrm{Cs}_{2} \mathrm{SnCl}_{6}$ using spark plasma sintering. Journal of American Ceramic Society 2018, 101, 2060-2065.

[2] Riley B.J., Vienna J.D., Frank S.M., Kroll J.O., Peterson J.A, Canfield N.I., Zhu Z.H., Zhang J.D., Crum J.V. Glass binder development for a glass bonded sodalite ceramic waste form. Journal of Nuclear Materials 2017, 489, 42-63.
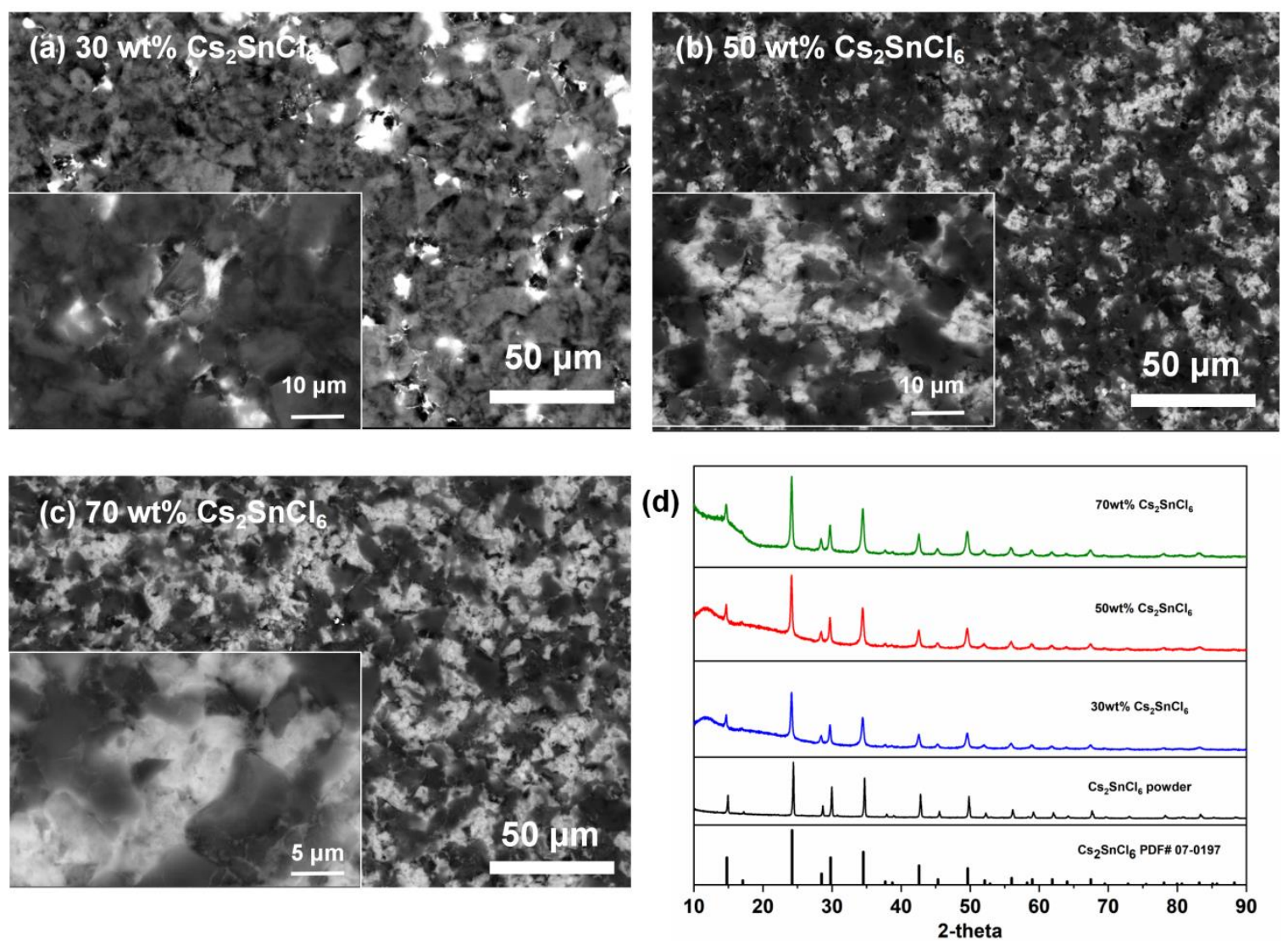

Figure s1. SEM images (BSE mode) of the polished surface of the as-sintered composite pellets (30 wt\%, 50 wt\% and $70 \mathrm{wt} \% \mathrm{Cs}_{2} \mathrm{SnCl}_{6}$-silica composites) (a, b and c) and corresponding XRD profiles as compared to the standard PDF card (d). 

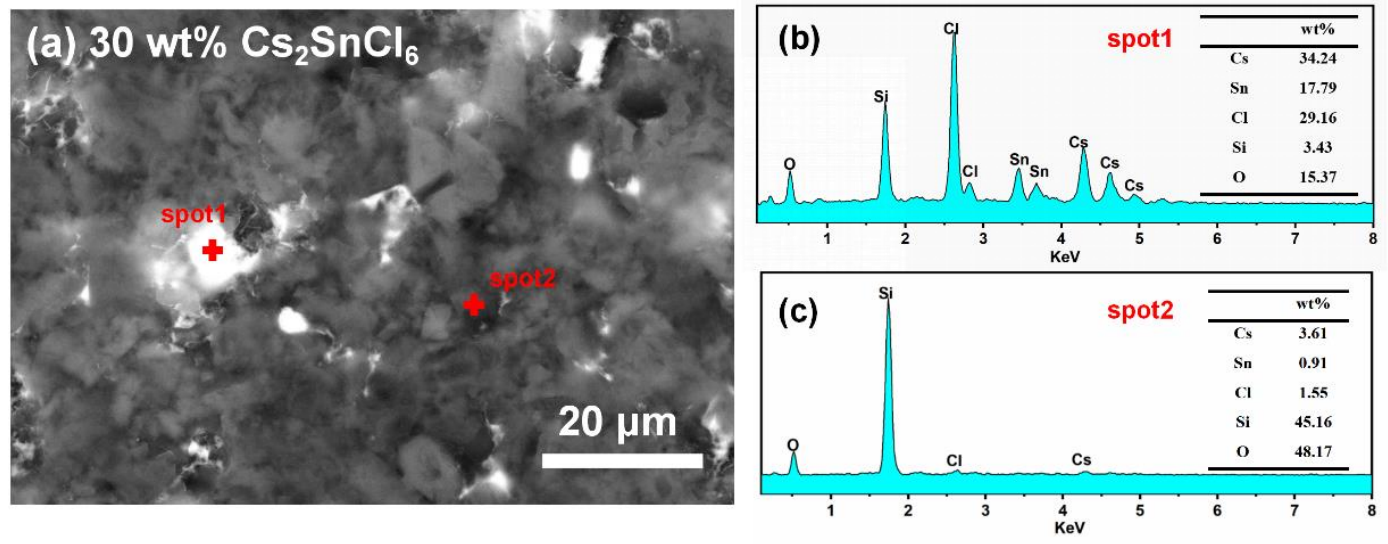

Figure s2. A SEM image (BSE mode) of the polished surface of the as-sintered composite pellet (30 wt\% $\mathrm{Cs}_{2} \mathrm{SnCl}_{6}$-silica) (a) and corresponding EDS spot analysis (b and c).
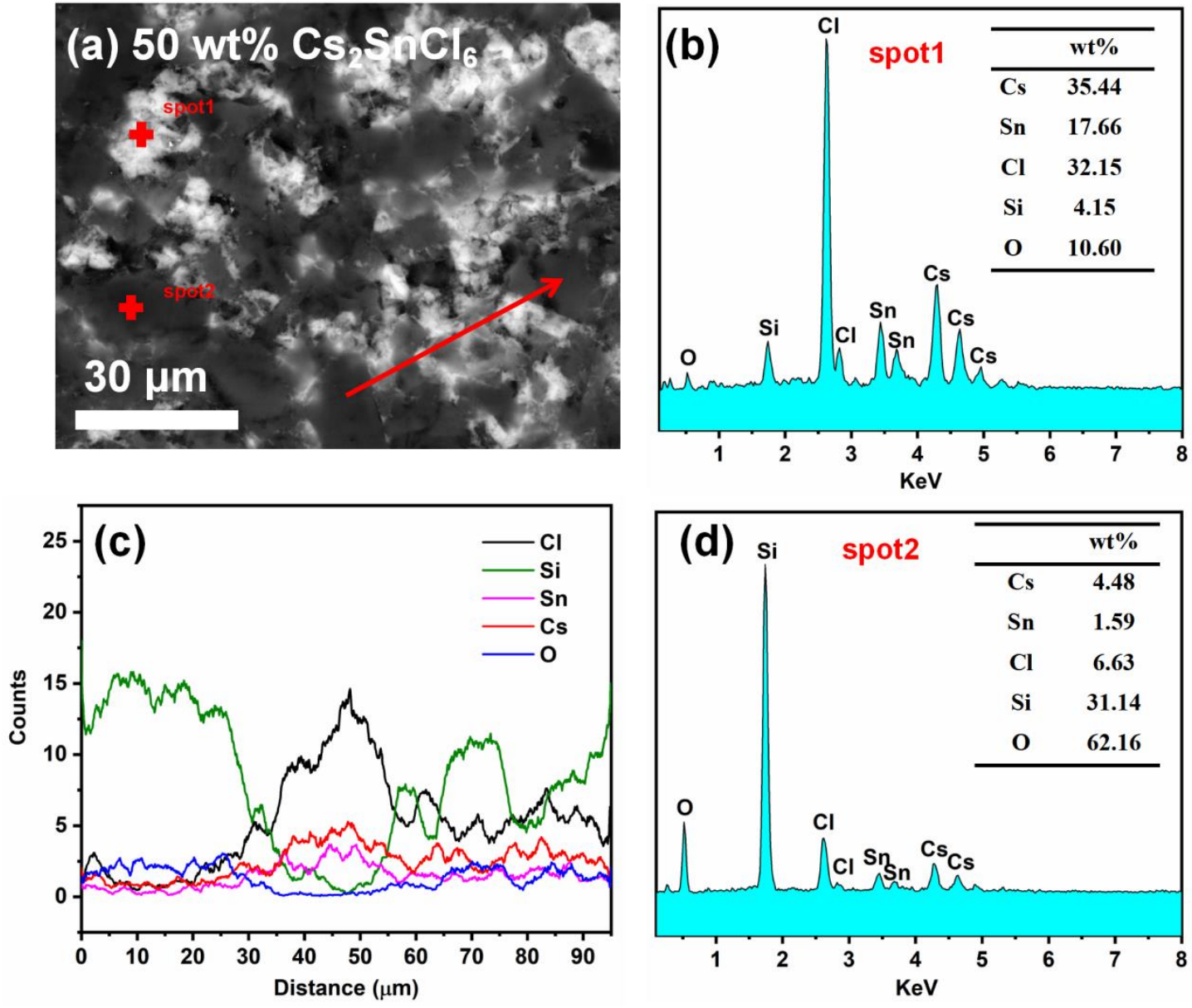

Figure s3. A SEM image (BSE mode) of the polished surface of the as-sintered composite pellet (50 wt\% $\mathrm{Cs}_{2} \mathrm{SnCl}_{6}$-silica) (a) and corresponding EDS spot analysis (b and d) and elemental line scanning across the $\mathrm{Cs}_{2} \mathrm{SnCl}_{6}$ and silica matrix dual phase (c). 

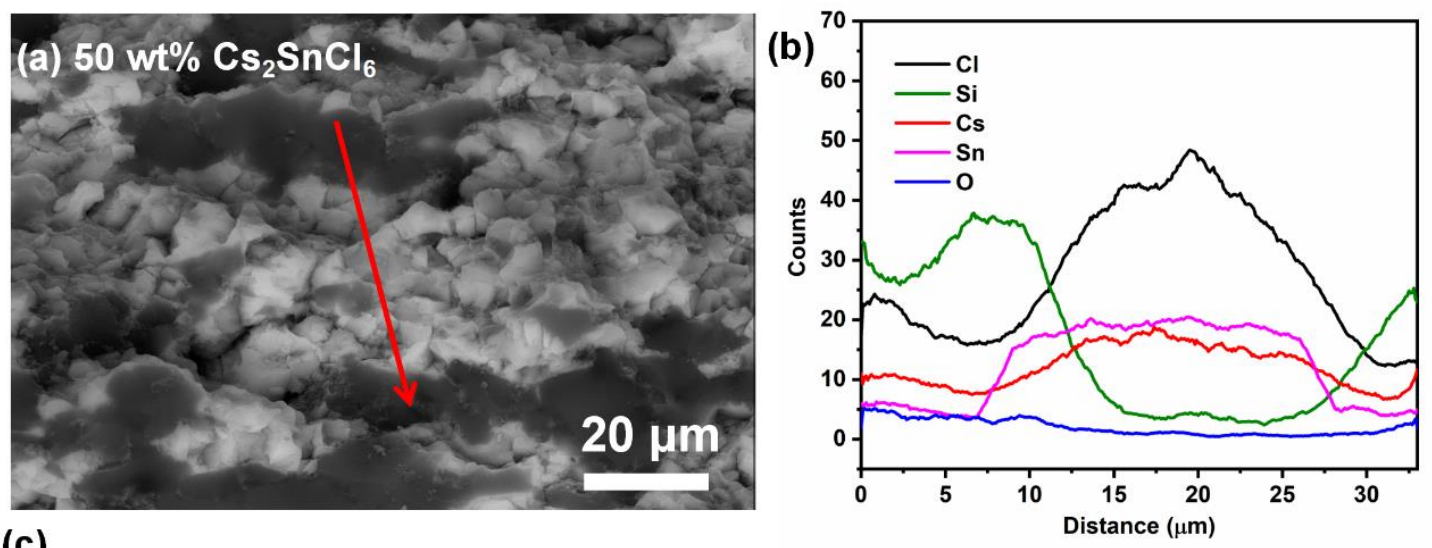

(c)
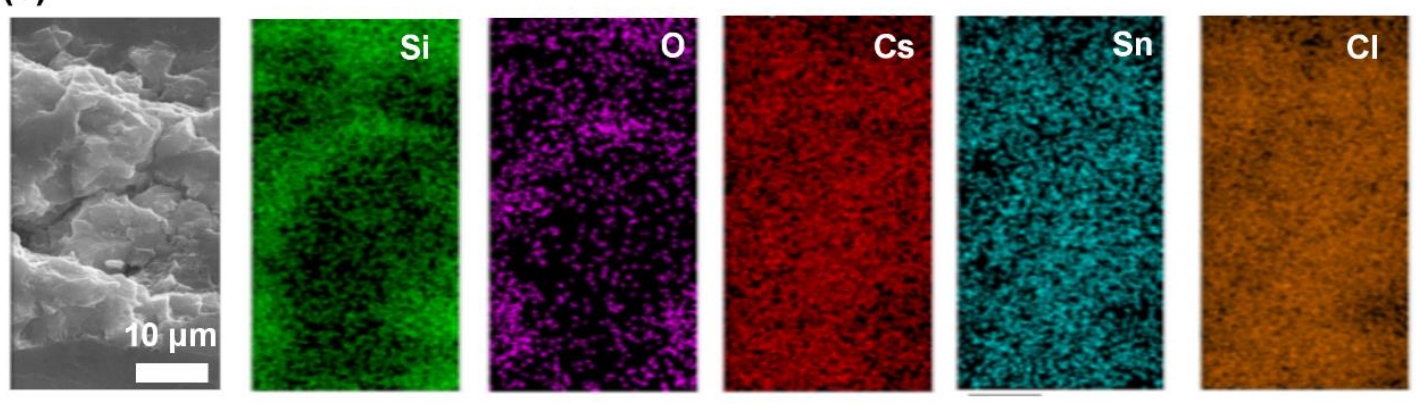

Figure s4. SEM images (BSE mode) of the fracture surface of the as-sintered composite pellet (50 $\mathrm{wt}_{\mathrm{C}} \mathrm{Cs}_{2} \mathrm{SnCl}_{6}$-silica) (a) and corresponding EDS line scanning analysis (b) and elemental mappings across the $\mathrm{Cs}_{2} \mathrm{SnCl}_{6}$ and silica matrix dual phase (c). 


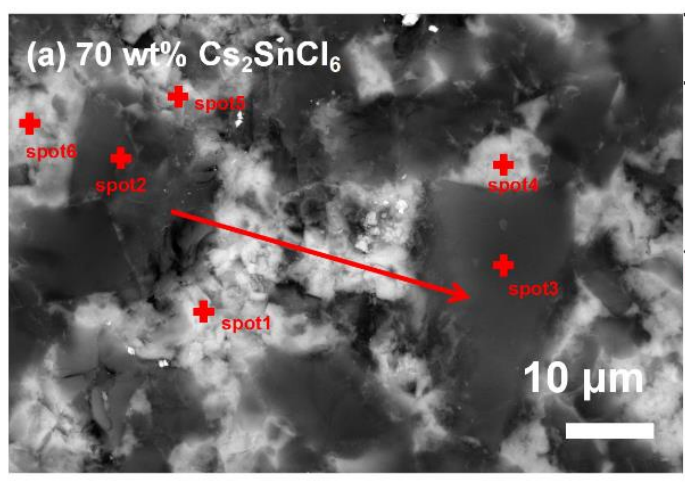

\begin{tabular}{ccccccc}
\hline & spot1 & spot2 & spot3 & spot4 & spot5 & spot6 \\
\cline { 2 - 7 } & $\mathbf{w t} \%$ & wt $\%$ & wt $\%$ & wt $\%$ & wt $\%$ & wt $\%$ \\
\hline Cs & 33.75 & 13.27 & 8.46 & 35.99 & 40.77 & 40.32 \\
Sn & 15.38 & 4.72 & 2.94 & 8.31 & 18.69 & 19.43 \\
Cl & 27.46 & 8.67 & 6.54 & 32.45 & 29.03 & 31 \\
Si & 7.77 & 29.43 & 30.01 & 4.94 & 4.77 & 3.7 \\
O & 15.64 & 43.91 & 52.05 & 8.31 & 16.74 & 5.55 \\
\hline
\end{tabular}
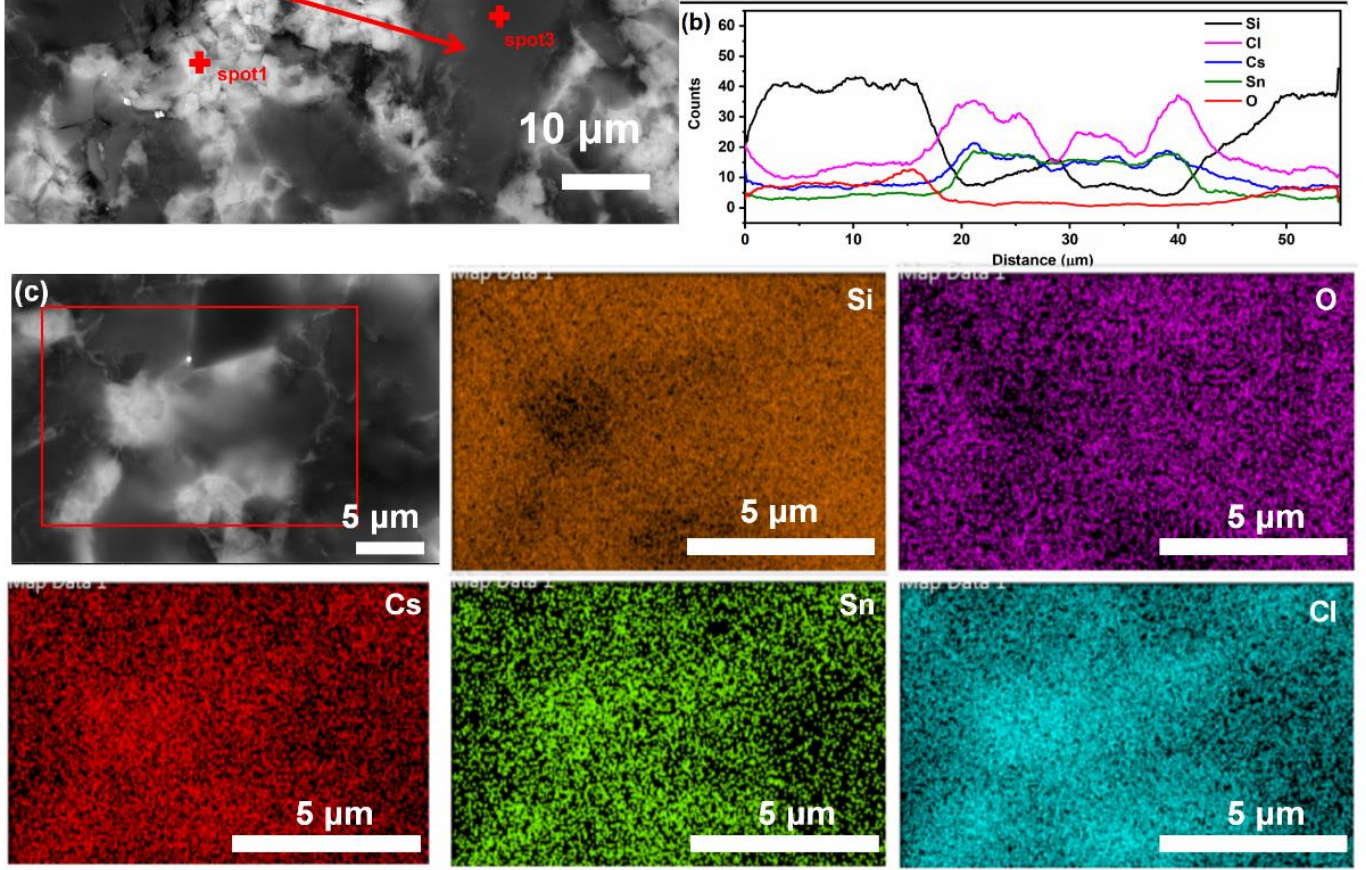

Figure s5. SEM images (BSE mode) of the polished surface of the as-sintered composite pellet (70 $w_{t} \% \mathrm{Cs}_{2} \mathrm{SnCl}_{6}$-silica) (a) and corresponding EDS spot analysis, elemental line scanning across the $\mathrm{Cs}_{2} \mathrm{SnCl}_{6}$ and silica matrix dual phase (b) and elemental mappings of the selected certain region (c). 

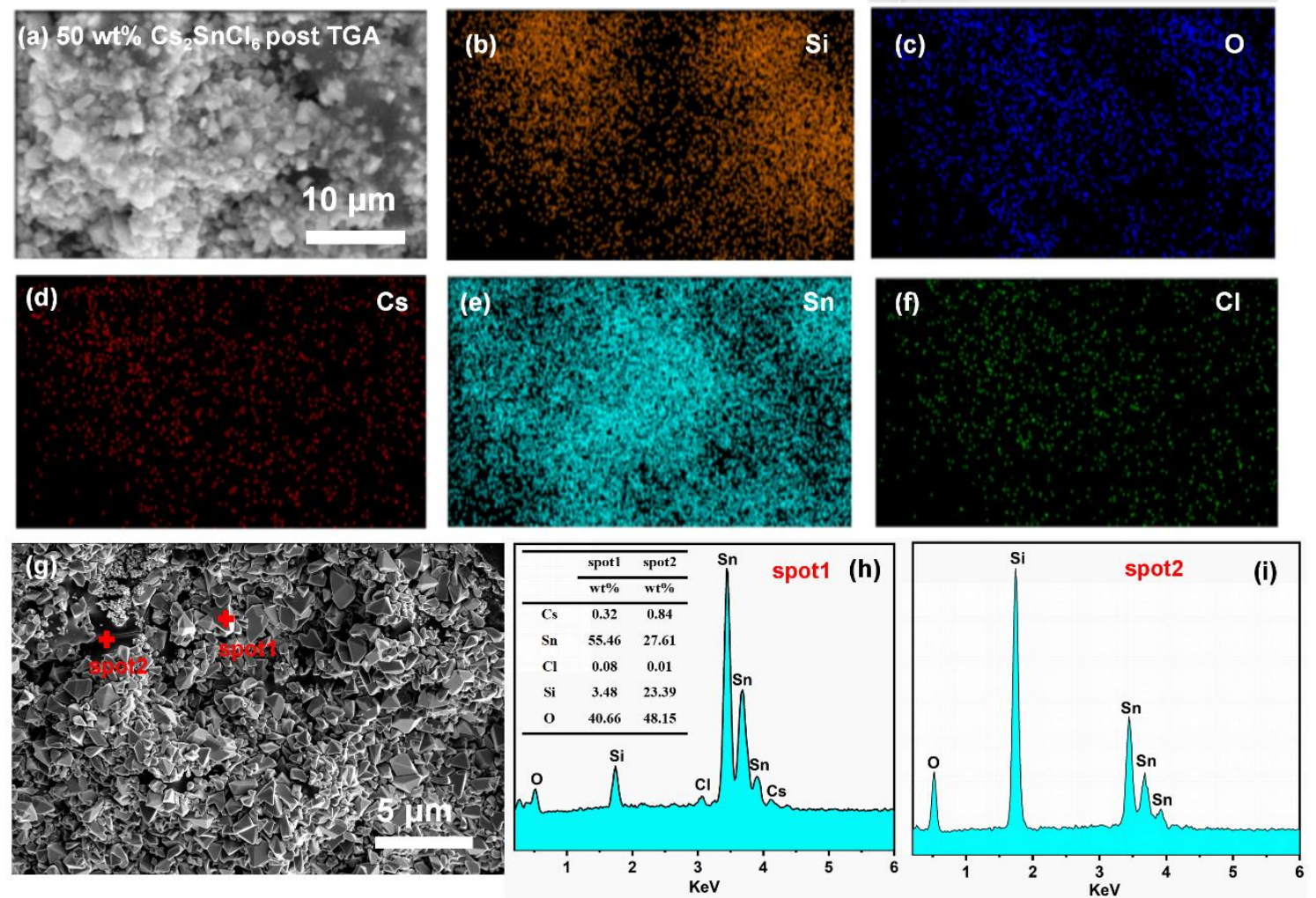

Figure s6. SEM images (SE2 mode) of the surface of the as-sintered composite pellets (50 wt\% $\mathrm{Cs}_{2} \mathrm{SnCl}_{6}$-silica) post thermal gravimetric analysis treatment (a) and corresponding elemental mappings (b-f), SEM image (SE2 mode) (g) and the corresponding spot semi-quantitative elemental analysis as shown in (h)-(i).
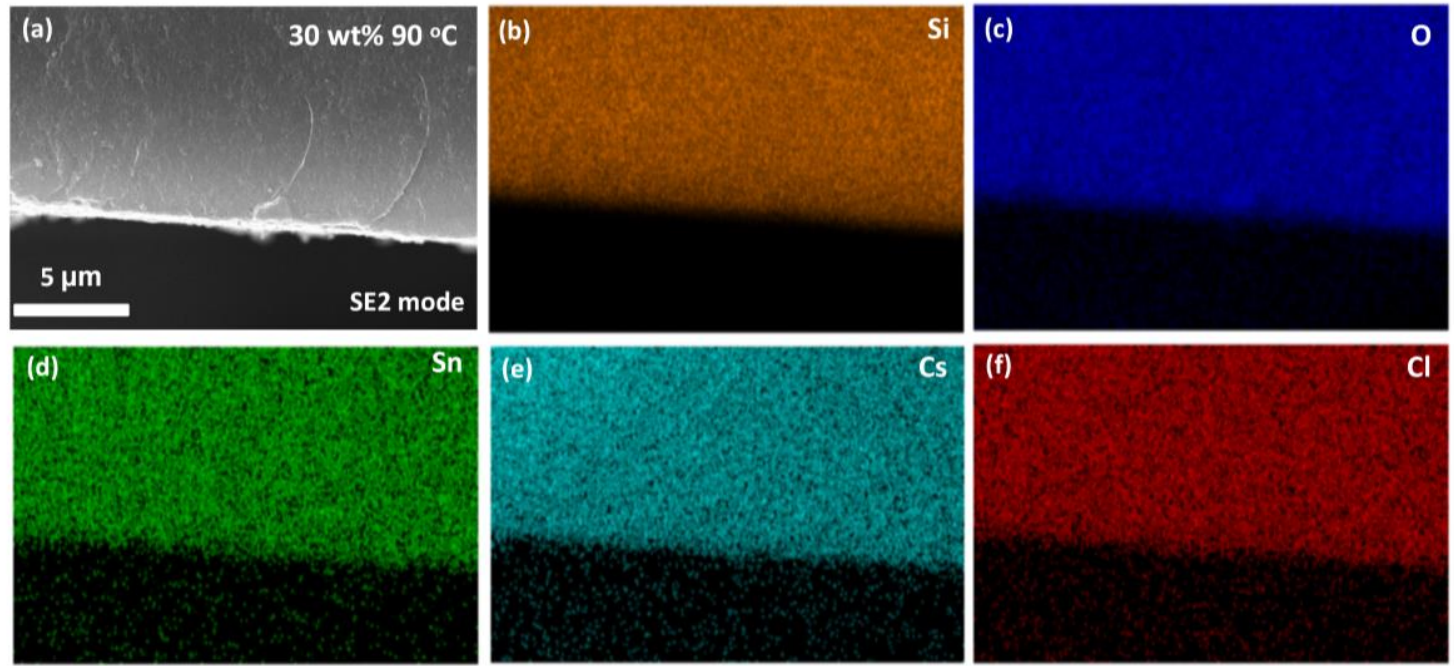

Figure s7. Surface morphology and elemental mappings of the cross-section of the $30 \mathrm{wt} \%$ 
composite leached at $90{ }^{\circ} \mathrm{C}$ for 14 days which does not show significant degradation.
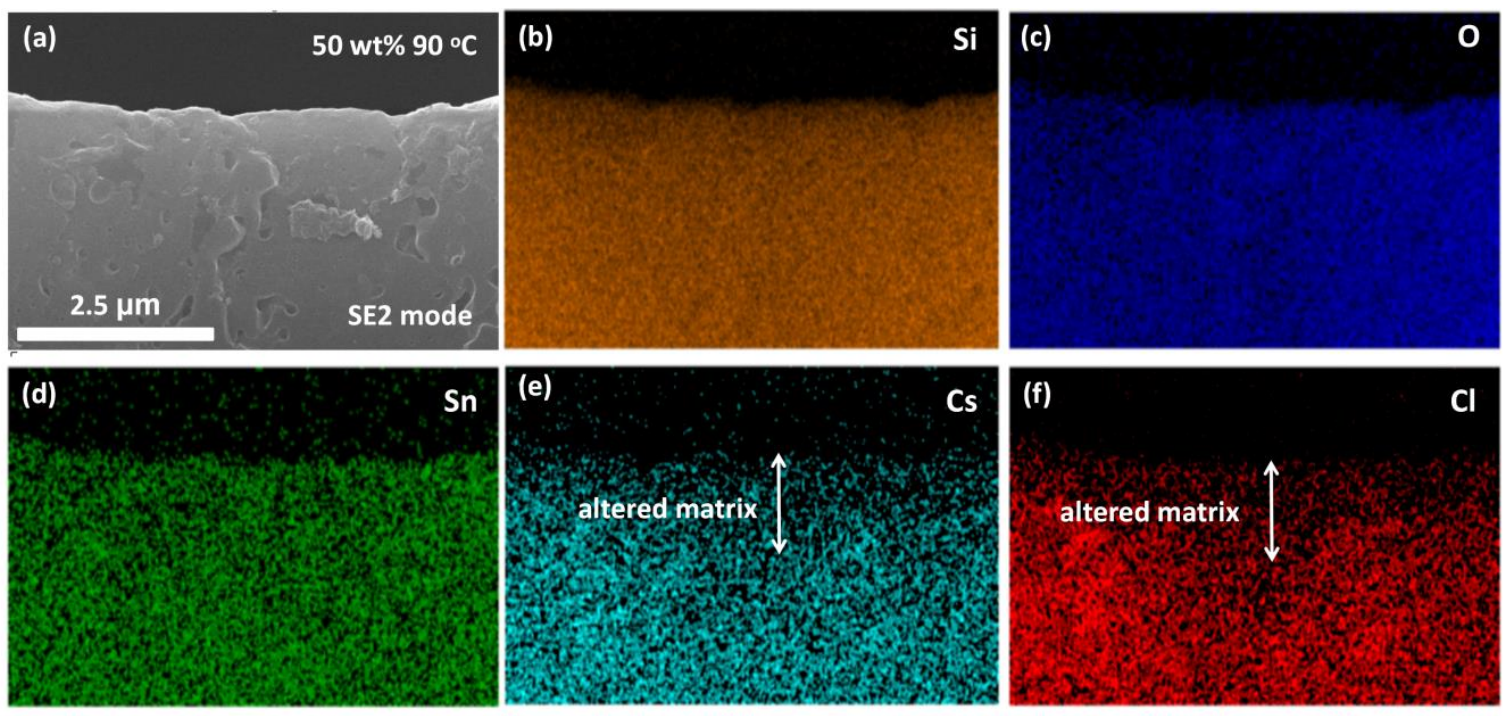

Figure s8. Surface morphology and elemental mappings of the cross-section of the $50 \mathrm{wt} \%$ composite leached at $90{ }^{\circ} \mathrm{C}$ for 14 days.

(a)

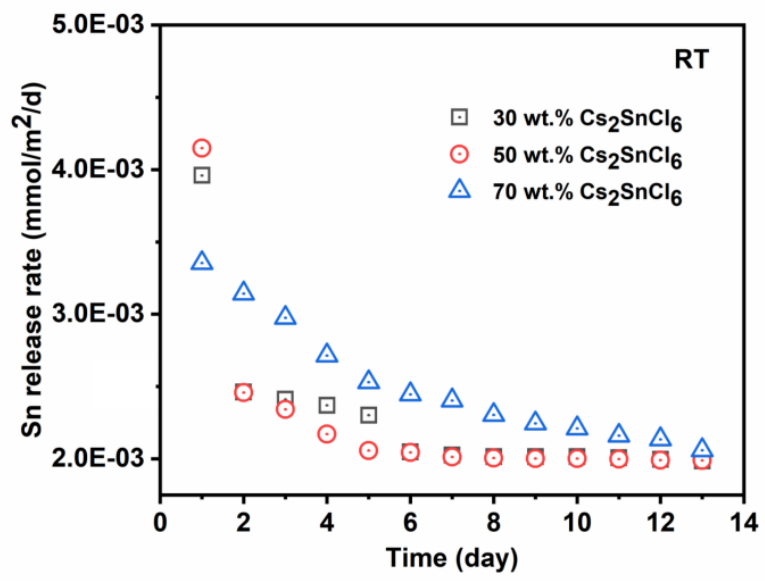

(b)

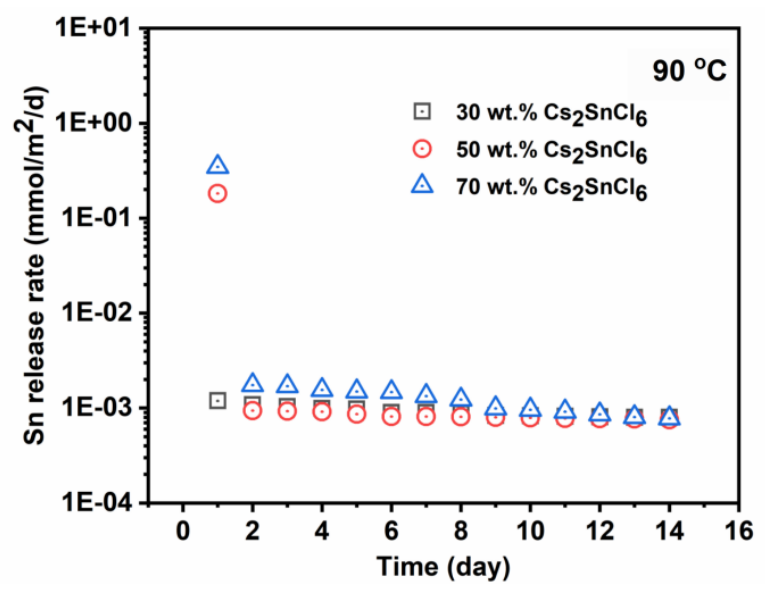

Figure s9. Semi-dynamic leaching results of the $\mathrm{Sn}$ of the $\mathrm{Cs}_{2} \mathrm{SnCl}_{6}$-silica composites: (a) leaching at room temperature; (b) leaching at $90^{\circ} \mathrm{C}$. 
(a)

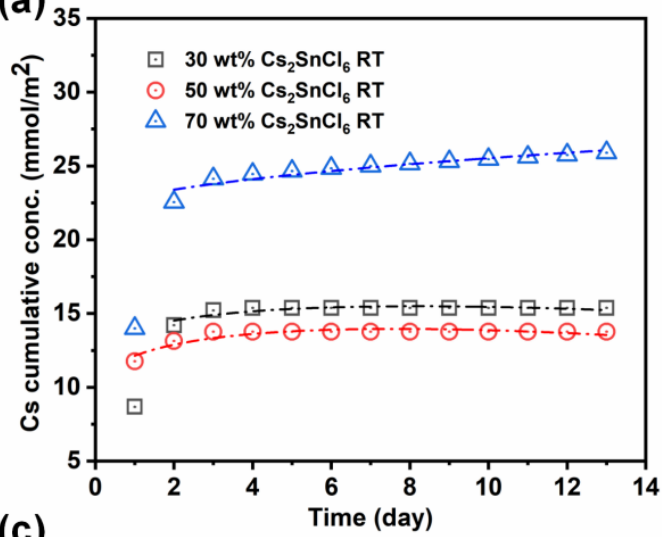

(c)

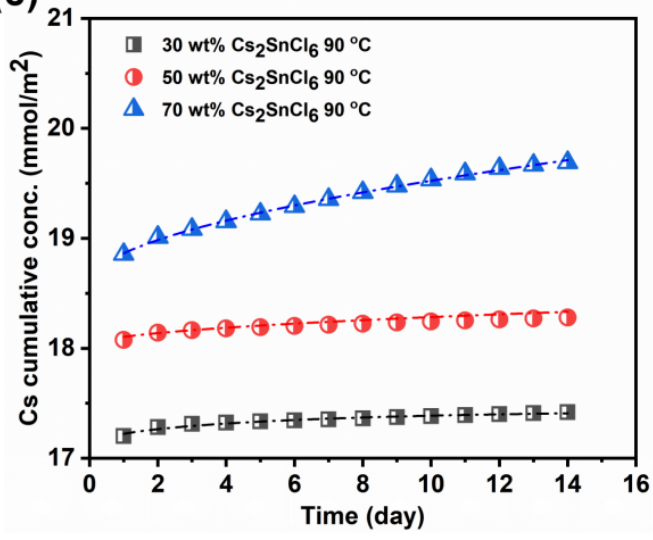

(b)

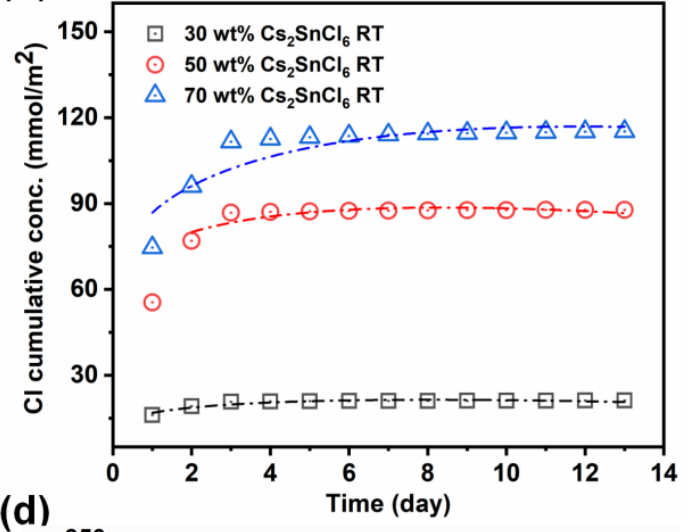

(d)

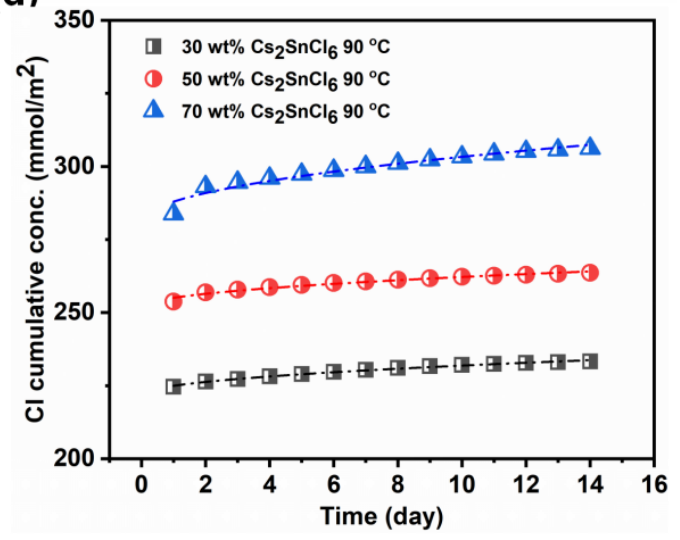

Figure s10. Cumulative element release mass curve fitted by the Cote's model; (a)-(c) Cs cumulative concentration at both room temperature and $90^{\circ} \mathrm{C}(\mathrm{b})-(\mathrm{d}) \mathrm{Cl}$ cumulative concentration at both room temperature and $90^{\circ} \mathrm{C}$. 
Table s1 Summary of various $\mathrm{Cs}$ and $\mathrm{Cl}$ immobilization methods and the corresponding leaching condition

\begin{tabular}{|c|c|c|c|c|}
\hline No. & description & Temp. $\left({ }^{\circ} \mathrm{C}\right)$ & leaching method & Reference \\
\hline 1 & $\begin{array}{c}\text { glass encapsulated calcium } \\
\text { phosphate ceramic }\end{array}$ & 40 & MCC-1 & Donald 2007 \\
\hline 3 & tellurite glass $(\mathrm{Cl})$ & 90 & C1285-02 & Riley 2012 \\
\hline 4 & calcium aluminum phosphate & 40 & C1285-02 & Schofield 2008 \\
\hline 5 & Cancrinite & 90 & C1285-02 & Riley 2012(JACS) \\
\hline 6 & Chlorapatite & 90 & MCC-1 & Vance 2012 \\
\hline 7 & chlorspodiosite & 90 & MCC-1 & Vance 2012 \\
\hline 8 & glass bonded sodalite & 90 & C1285-02 & Ebert 2005 \\
\hline 9 & $(\mathrm{Ba}, \mathrm{Cs})(\mathrm{Al}, \mathrm{Ti})_{2} \mathrm{Ti}_{6} \mathrm{O}_{16}(\mathrm{Cs})$ & 90 & MCC-1 & Carter (2002) \\
\hline 10 & $\begin{array}{c}\text { Hollandite } \\
\left(\mathrm{BaCs}_{0.28} \mathrm{Fe}_{0.82} \mathrm{Al}_{1.46} \mathrm{Ti}_{5.72} \mathrm{O}_{16}\right)\end{array}$ & 90 & MCC-1 & Angeli (2008) \\
\hline 11 & Borosilicate $(\mathrm{Cs})$ & 90 & MCC-1 & Lee (2006) \\
\hline 12 & Portland cement (Cs) & RT & GOST 29114-91 & Ojovan (2011) \\
\hline 13 & $\begin{array}{c}\text { Struvitec }(\mathrm{CsCl}) \text {-borosilciate } \\
\text { matrix }\end{array}$ & RT & ANS 16-1 & Wagh (2016) \\
\hline 14 & Cs-pollucite $+\mathrm{SiO}_{2}(\mathrm{Cs})$ & 97 & MCC-5 & Yanagisawa(1987) \\
\hline 15 & HIPed tailored hollandite $(\mathrm{Cs})$ & 90 & MCC-1 & Carter (2009) \\
\hline 16 & $\mathrm{Cs}_{0.21} \mathrm{Na}_{0.12} \mathrm{~W}_{0.07} \mathrm{Mo}_{0.02} \mathrm{O}_{3}(\mathrm{Cs})$ & 150 & MCC-1 & Luca (2004) \\
\hline 17 & $\mathrm{Ba}_{0.85} \mathrm{Cs}_{0.26} \mathrm{Al}_{1.35} \mathrm{Fe}_{0.77} \mathrm{Ti}_{5.9} \mathrm{O}_{16}(\mathrm{Cs})$ & 95 & UndefinedTest & Muresan (2011) \\
\hline 17 & $\mathrm{Ba}_{0.67} \mathrm{Cs}_{0.53} \mathrm{Ga}_{2.04} \mathrm{Ti}_{6} \mathrm{O}_{16}(\mathrm{Cs})$ & 90 & C1285-02 & Zhao (2018) \\
\hline 18 & Iron phosphate glass (DPF-1h) & 90 & C1308 & Ebert (2018)A \\
\hline 19 & Iron phosphate glass (DPF-P1) & 90 & C1308 & Ebert (2018)B \\
\hline
\end{tabular}




\begin{tabular}{|c|c|c|c|c|}
\hline 20 & $\begin{array}{l}\text { Two-phase glass bonded sodalite } \\
\text { (CWF) }\end{array}$ & 90 & C1308 & Ebert (2015)A \\
\hline 21 & $\begin{array}{c}\text { Advanced two-phase glass bonded } \\
\text { sodalite (ACWF-N3) }\end{array}$ & 90 & C1308 & Ebert (2015)B \\
\hline 22 & $\mathrm{PbTeO}_{2}$ TEer10A & 90 & C1308 & Ebert (2015)C \\
\hline 23 & Tellurite glass (Cs) & 90 & C1308 & Riley (2020) \\
\hline 18 & $\begin{array}{c}\mathrm{Cs}_{2} \mathrm{SnCl}_{6} \text {-silica composite }(30,50, \\
70 \mathrm{wt} \%)\end{array}$ & $\mathrm{RT}, 90$ & C1308 & this work \\
\hline
\end{tabular}

Table s2 Summary of various $\mathrm{Cs}$ and $\mathrm{Cl}$ immobilization capacity and normalized long-term rate

\begin{tabular}{|c|c|c|c|c|c|c|}
\hline $\begin{array}{c}\text { No } \\
\text {. }\end{array}$ & description & $\begin{array}{c}\text { Cl loading } \\
(\mathbf{w t} \%)\end{array}$ & $\begin{array}{c}\text { Cs } \\
\text { loading } \\
(w t \%)\end{array}$ & $\begin{array}{c}\text { Normalized } \\
\text { long-term rate } \\
\left(\mathbf{m g N} / \mathbf{m}^{2} / \mathbf{d}\right)(\mathrm{Cl})\end{array}$ & $\begin{array}{c}\text { Normalized } \\
\text { long-term rate } \\
\left(\mathbf{m g N} / \mathrm{m}^{2} / \mathbf{d}\right)(\mathrm{Cs})\end{array}$ & Reference \\
\hline 1 & $\begin{array}{l}\text { glass encapsulated } \\
\text { calcium phosphate } \\
\text { ceramic }\end{array}$ & 4 & & 3.4 & & Donald (2007) \\
\hline 3 & tellurite glass $(\mathrm{Cl})$ & 5 & & 528 & & Riley (2012) \\
\hline 4 & $\begin{array}{l}\text { calcium aluminum } \\
\text { phosphate }\end{array}$ & 6.8 & & 170 & & $\begin{array}{l}\text { Schofield } \\
\text { (2008) }\end{array}$ \\
\hline 5 & Cancrinite & 2.6 & & 140 & & $\begin{array}{c}\text { Riley } \\
\text { 2012(JACS) }\end{array}$ \\
\hline 6 & Chlorapatite & 6.5 & & 84 & & Vance (2012) \\
\hline 7 & chlorspodiosite & 2.2 & & 875 & & Vance (2012) \\
\hline 8 & glass bonded sodalite & 2 & & 1200 & & Ebert (2005) \\
\hline 8 & $\begin{array}{c}\text { Two-phase glass bonded } \\
\text { sodalite }(\mathrm{CWF})\end{array}$ & 4.6 & 0.31 & 800 & 400 & Ebert (2015)A \\
\hline
\end{tabular}


Advanced two-phase glass

9 bonded sodalite (ACWF-

$\begin{array}{lll}6.37 & 0.44 & 20000\end{array}$

3000

Ebert (2015)B

N3)

$10 \mathrm{PbTeO}_{2}$ glass with ER salt

7.2

0.13

15000

830

Ebert (2015)C

$9(\mathrm{Ba}, \mathrm{Cs})\left(\mathrm{Al}, \mathrm{Ti}_{2}\right)_{2} \mathrm{Ti}_{6} \mathrm{O}_{16}(\mathrm{Cs})$

1.66

5

Carter (2002)

$10\left(\mathrm{BaCs}_{0.28} \mathrm{Fe}_{0.82} \mathrm{Al}_{1.46} \mathrm{Ti}_{5.72} \mathrm{O}\right.$

0.96

5

Angeli (2008)

16)

11 Borosilicate (Cs)

10

30

Lee (2006)

12 Portland cement (Cs)

1.6

700

Ojovan (2011)

13 Struvitec $(\mathrm{CsCl})-$

1.58

266

Wagh (2016)

14 Cs-pollucite+ $\mathrm{SiO}_{2}(\mathrm{Cs})$

0.49

3150

Yanagisawa(19

87)

15 HIPed tailored hollandite

6

$69 \quad$ Carter (2009)

(Cs)

$16 \mathrm{Cs}_{0.21} \mathrm{Na}_{0.12} \mathrm{~W}_{0.07} \mathrm{Mo}_{0.02} \mathrm{O}_{3}$

(Cs)

10

7

Luca (2004)

${ }_{17} \mathrm{Ba}_{0.85} \mathrm{Cs}_{0.26} \mathrm{Al}_{1.35} \mathrm{Fe}_{0.77} \mathrm{Ti}_{5.9}$

0.92

0.08

Muresan

$\mathrm{O}_{16}(\mathrm{Cs})$

(2011)

$\mathrm{Ba}_{0.67} \mathrm{Cs}_{0.53} \mathrm{Ga}_{2.04} \mathrm{Ti}_{6} \mathrm{O}_{16}$

(Cs)

7.8

1400

Zhao (2018)

Iron phosphate glass

(DPF-1h) (Cs)

0.25

5680

Ebert (2018)A

Iron phosphate glass

(DPF-P1) (Cs)

0.25

990

Ebert (2018)B

Tellurite glass (Cs)

4.5

9240

Riley (2020)

18

$\mathrm{Cs}_{2} \mathrm{SnCl}_{6}$-silica composite

$11 \quad 13.4$

23.6

3.46

this work 


\begin{tabular}{cccccc}
$19 \begin{array}{c}\mathrm{Cs}_{2} \mathrm{SnCl}_{6} \text {-silica composite } \\
(50 \mathrm{wt} \%)\end{array}$ & 18.3 & 22.3 & 32.3 & 3.51 & this work \\
$20 \begin{array}{c}\mathrm{Cs}_{2} \mathrm{SnCl}_{6} \text {-silica composite } \\
(70 \mathrm{wt} \%)\end{array}$ & 25.5 & 31.2 & 54 & 9.06 & this work \\
\hline
\end{tabular}

